\title{
Development of Cost Optimized Horizontal Gel Electrophoresis Running Unit for Developing Countries (Nepal)
}

\author{
Sajesan Aryal, Aroj Hada, Sanjay Hamal, Abhishek Prajapati, Paras Mani Timilsina,
}

Sandeep Adhikari *

Department of Biotechnology, Kathmandu University

\begin{abstract}
The use of expensive lab techniques has left many high schools and even university students unacquainted with the basic experimental procedures and protocols in developing country including Nepal. Horizontal gel electrophoresis is one of the expensive protocols, which every student in the laboratory may not get an equal chance to access individually. However, this technique, being indispensable and inevitable in molecular biology principles, is of abounding importance for students to be familiar with. Thus, realizing its importance, we present an extremely simple and inexpensive design of gel-electrophoresis unit, which emulates electrophoresis analysis with the use of nichrome and aluminum wires as a substitute for platinum wires, together with daily used plastic materials. Because of these factors, the approximate cost of unit design is significantly reduced to an amount of 10 USD. The efficiency of the substitute wires was confirmed and it resulted in satisfactory data characterized by good resolution of the DNA fragments. The inexpensive nature, good results and simplicity of the device make it an ideal unit for teaching and learning in developing countries.

Keywords: Horizontal Gel Electrophoresis, Molecular biology, Nepal, Nichrome.

*Corresponding Author
\end{abstract}

Email: acsandeep2014@gmail.com

\section{Introduction}

In graduate courses and research environment, the use of gel electrophoresis seems to be an expensive technique. The main concern lies on the fact that many developing countries limit their courses on theoretical concepts unaccompanied by experimental practices due to cost ineffectiveness. Learning theoretical science concepts and lack of opportunity to apply the very concept on the real world only provides an abstract vignette of knowledge for the students. "The core reason that may underlie upon the fact that many developing countries emphasizes teaching theory in secondary, higher secondary and sometimes even in undergraduate courses, is the absence of expensive laboratory equipment. "( Ens et al, 2012) [1]. Gel electrophoresis is expensive equipment which many colleges and even some universities of developing countries cannot afford in adequate numbers so that all the students can equally access them. Thus, this article proposes an inexpensive and result oriented simpler design alternative for macromolecule separation replacing the expensive equipment.

Gel electrophoresis is one of the most widely used molecular biology technique for the separation and analysis of the macromolecule (DNA, RNA, and proteins) fragments based on their size and charge. The technique is based on the use of electric field to separate different sized macromolecule fragments in a porous gel matrix. The electric field is applied in such a way that one end of the gel has a positive charge and another end has a negative charge. As macromolecules like DNA and RNA have a negative charge due to the presence of phosphate groups, they tend to move from the negative pole to the positive pole of the gel under the application of electric field. [2] Finally, after the samples have been separated using gel electrophoresis technique, bands representing molecules of different sizes can be detected.

Hereby, we designed a similar electrophoresis unit which emulates the original laboratory gel electrophoresis unit, constructed with readily available inexpensive materials and showed its efficacy with the electrophoresis of DNA samples as described below. The test and analysis of the protocol were performed at molecular biology laboratory at Kathmandu University (KU), Dhulikhel, Nepal. The major focus during the project was given on the key element of construction of electrophoresis unit and 
preparation of gels and buffers, which generally remains invisible to the students during the teaching process. Along with these parts, diagnostic tests were repeatedly performed to check the durability of the protocol and the quality of result obtained by visualizing the DNA bands under UV radiation.

\section{Materials and Methods}

Two micro-wave safe plastic boxes of (16.8 X 11.2) $\mathrm{cm}$ and $(10.75 \times 11) \mathrm{cm}$ dimension were bought from a local plastic store. Also, a Plexi-glass of dimension $(11 \times 11) \mathrm{cm}$ was bought from a glassware store. A $30 \mathrm{~cm}$ measuring scale was bought from a local stationary. Nichrome, aluminum and two multimeter wire one black and another red in color was bought from a local electric store.

\section{Instrumentation}

A micro-wave safe plastic box of $(16.8 \times 11.2) \mathrm{cm}$ dimension was used as the electrophoresis tank. A Plexiglass of dimension $(11 \times 11) \mathrm{cm}$ was attached to the bottom of this tank leaving some spaces on the both side of the plastic box so that it mimics the platform where gel-box is supposed to sit as in laboratory equipment.

Two kinds of electrode arrangement were tested. First, Double wounded nichrome wire as both cathode and anode. Second, Double wounded nichrome as cathode and triple wounded aluminium as anode. Variation of electrodes in anode was brought to test the durability and effectiveness of the two different wires; nichrome and aluminium. This was done so, because the electrode at anode corroded at higher rate in comparision with the electrode at cathode.

For the gel holding tray, a suitable plastic box of dimension $(10.75 \times 11) \mathrm{cm}$ was fitted in the buffer tray. Leaving $1 \mathrm{~cm}$ from the bottom of the gel holding tray, the entire tray was cut horizontally. Again, the two smaller opposite ends of the gel holding tray were cut vertically so that it mimics the shape and form of the standard gel box used in the laboratory.

For the gel comb, a ruler of considerable thickness was chosen. Each groove of $0.5 \mathrm{~cm}$ with $0.5 \mathrm{~cm}$ gap in between was made. Ruler was cut on either side to make it exactly fit and enter inside the buffer tray. It was cut in such a way that the teeth of the ruler do not touch the bottom of the gel holding tray.
Two multimeter wires were fitted in the lid of electrophoresis unit, by drilling the extreme corners but of the same side of the lid and the wires were fitted into the drilled holes. The tip of both the wires was cut and joined with two

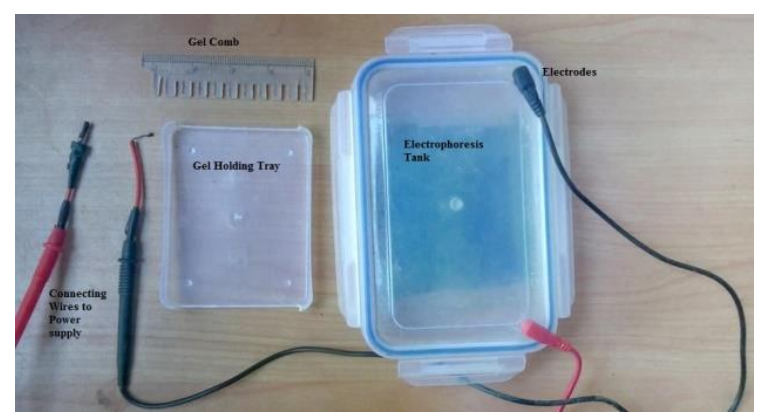

alligator leads which fit in the available voltage regulator.

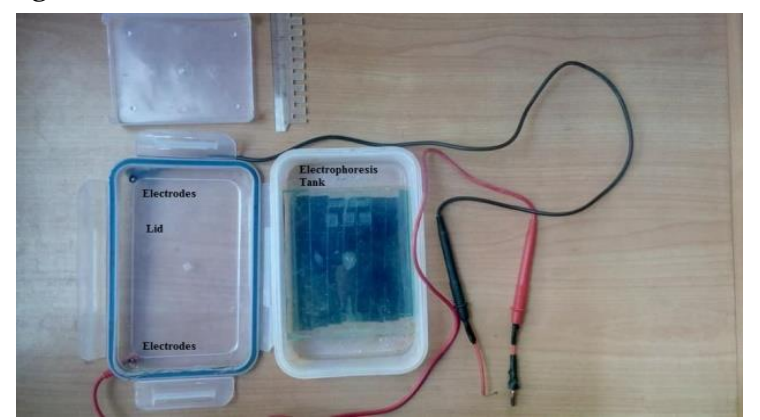

Figure 1: Overall instrumentation of the electrophoresis unit

\section{Gel and Running Buffer}

$1 \%$ agarose gel was used in all of the processes. Before the agarose solution was polymerized into gel, $0.5 \mu \mathrm{g} / \mathrm{ml}$ of Ethidium Bromide (EtBr) was added after gel reached $65^{\circ} \mathrm{C}$ and stirred. Ethidium Bromide acts as an intercalating agent between the two strands so that upon visualization of the DNA bands in the UV it can be properly viewed. $[3,4]$

The running buffer was prepared according to standard laboratory practices (either 0.5X TBE or TAE). The DNA samples were loaded in the wells of the gel loading tray and the tray was immersed into the buffer solution completely. Then, the lid of the electrophoresis tray was closed so as to complete the circuit and the voltage was applied. Care was taken that the wells on the gel were on cathode (negative terminal) so that on applying electric field DNA could move towards the anode (positive terminal). A constant voltage was set up and a time of $120 \mathrm{~min}$ for all test runs. The current was applied. After the designated time, the gel was removed and then visualized under the UV radiation and the DNA bands were observed. [5] 
Result and Discussion

Efficiency of the electrophoresis unit: For nichrome as both cathode and anode

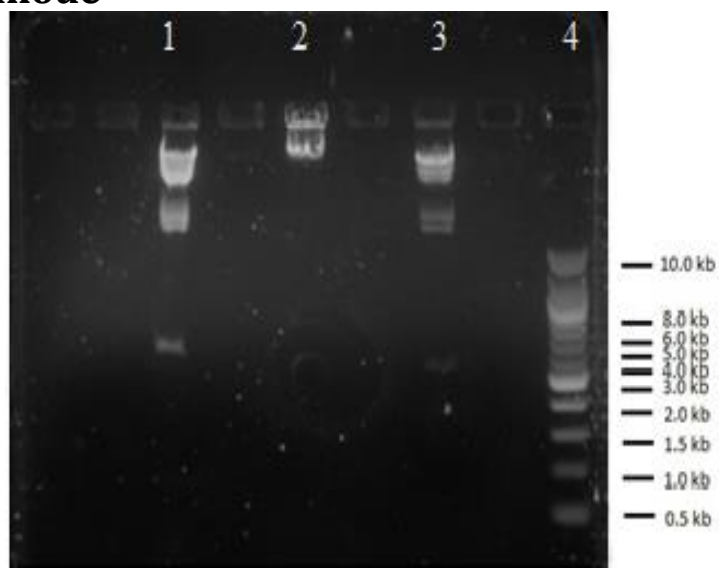

Figure 2A: DNA bands from an agarose (1\%) gel with TBE buffer run for $55 \mathrm{~V}$ for 2 hours using nichrome as both the electrodes.
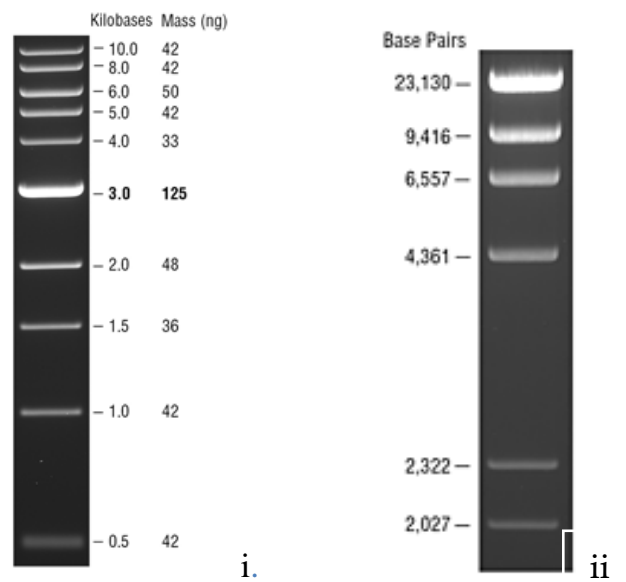

Figure: 2B: Gel image of (i) $1 \mathrm{~kb}$ DNA iadier and (ii) Lambda DNA/HINDIII obtained from NEB (Gel run on $1 \%$ agarose concentration)

Firstly, our unit was run using standard DNA samples of $\lambda$ DNA Hind III digest, $\lambda$ DNA, $\lambda$ DNA Hind III digest and $1 \mathrm{~kb}$ DNA ladder, in the order from left to right, as shown in Figure 2A. All the samples were standards obtained from NEB (New England Biolabs).

The DNA ladder in lane 4 was separated into 10 fragments identical to the standard gel image obtained from NEB [6]. The Lambda DNA digested by HindIII in lane 3 separated into 5 fragments which contradicted with the standard NEB gel image. This may have happened because the time of the run was not enough for the separation to complete. The $1 \mathrm{~kb}$ DNA ladder in lane 4 separated into 10 fragments as shown in Figure: $2 \mathrm{~A}$.

Comparing the two images (Figure 2A and Figure 2B), it is clear that the band separation of each sample in both the runs were similar (the standard obtained from NEB and the one obtained from our unit). From these results, we are able to say the efficiency of migration of DNA samples is quite satisfactory while using nichrome electrodes in place of platinum electrodes.

\section{DNA Ladder Standard Curve}



Figure 2C: DNA ladder standard calibration curve (Log10Fragment size (kb) vs. Migration (mm)) obtained from the data interpreted from fig (1a). Distances were measured from image processing software: Image [8]

\section{For nichrome cathode and aluminum anode}

Secondly, the unit was run using nichrome at cathode and aluminum at anode. Samples of lambda DNA and lambda DNA Hind III digest (from left to right) were used and the following gel image was obtained (Figure 3A).

The Lamda DNA digested by HindIII in lane 2 separated into 5 distinct bands as shown in the gel image below (Figure 3A). A fragment of the Lambda DNA/HindIII digest was not clearly observed. This may have happened due to not enough run time or low intensity of the band. All the samples were standards obtained from NEB (New England Biolabs).

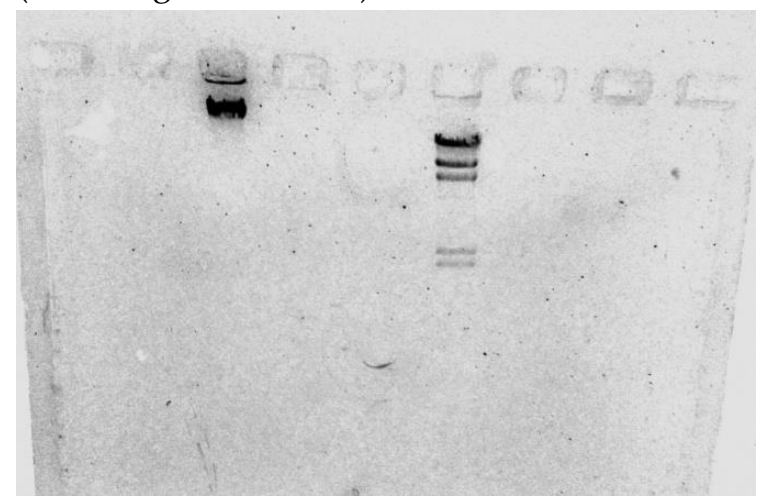

Figure 3A: $\lambda$ DNA/HindIII bands from an agarose (1\%) gel with TBE buffer run for $55 \mathrm{~V}$ for 2 hour in apparatus using nichrome as cathode and aluminum as anode.

Comparing the two images (Figure 3A and Figure 2B (ii)), it is clear that the band separation of each 
sample in both the runs were similar and differ by only one band (the standard obtained from NEB and the one obtained from our unit).

From these results, we are able to say the efficiency of migration of bands is quite satisfactory while using nichrome at cathode and aluminum at anode in place of platinum electrodes.

\section{DNA/HINDIII Standard Curve}



Figure 3B: Lambda DNA/Hind III standard curve (Fragment size $(\mathrm{kb})$ vs. Migration $(\mathrm{mm})$ ) obtained from the data interpreted from figure $\mathbf{2 B}$.

From these observations, we verified that use of nichrome or aluminum wires as electrodes provide similar resolution to that of commercially available platinum wired units.

\section{Analysis of Instrument's durability:}

Electrode materials, Aluminum and nichrome could be used for a total runtime of $6-8$ hours. The use of nichrome wires as both the electrodes allowed a run of 6-8 hours after which significant deterioration of the nichrome wires at anode was observed while the metal wire at cathode remained intact. Degradation of anode was visible as decrement of the wire diameter.

Therefore, efficiency of another metal (Aluminum) at anode was checked. Although corrosion and pitting of aluminum at anode as white flocculent material was observed during electrophoresis, electrodes remained intact for normal runs of about 3 times (6 hours). Aluminum wire at anode reacted with components of the buffer electrolyte during the run and formed a protective layer in the form of white flocculent material. The material did not interfere with the run as seen by the gel image (Figure 2A) [6].

Then another thrice wounded aluminum or chromium wire can be fitted in an L-shaped manner easily as described in instrumentation protocol.
In contrast, nichrome at cathode seemed very effective as it did not show any signs of corrosion even for a long run over 8 times (16 hours). These data of wire durability were obtained after triplicate experimental runs.

Variation of electrodes in anode was brought to test the durability and effectiveness of the two different wires; nichrome and aluminium. This was done so because the electrode at anode corroded at higher rate in comparision with the electrode at cathode. This occurs due to the oxidation of anode. Electons travel from anode to cathode. Hence metal of anode is continuously converted to ion and anode is pitted and consumed continuously (corrosion).

This is the reason for using platinum as electodes. Platinum is a transition metal in Group 10 (VIII B) of the periodic metal. They are noble metals and hence are relatively non-reactive. Platinum does not react with air, water etc and does not corrode at any temperature. Hence platinum is very suitable to use as electrodes. However its price is very high i.e. 930 USD/ozt. The cost of nichrome wire is $2.29 \mathrm{USD} / \mathrm{kg}$ and that of aluminium is 0.0582 USD/ozt [7].

Micro-wave safe plastic boxes were chosen over glass to ensure that the heat produced from the electrodes would not damage the interiors of the box at a low price. The interior layer of the plastic box was not damaged even after the overall experiment run. Thus, it was concluded the use of micro-wave safe plastic box, which are easily available at relatively low cost can effectively be used in the protocol.

Plexiglass at the bottom of the gel box also didn't show any signs of damage during the overall period of run. Also, the wires and alligator clips resulted as quite good alternatives in the design of the unit.

\section{Cost Estimate and Comparison:}

Table 1: cost estimation of the units

\begin{tabular}{lc}
\hline Apparatus & Cost (USD) \\
\hline Microwave safe box & 5 \\
Plexi-Glass & 2 \\
Multimeter Wires & 2 \\
Nichrome \& Aluminum Wires & 1 \\
\hline Total & 10 (approx.) \\
\hline The electrophoresis unit was made with a goal to \\
reduce the cost of the overall & unit so that \\
universities and colleges in developing countries \\
are able to provide quality practical education to
\end{tabular}


the students with less expense. The detailed cost estimation of the unit is Table 1.

The cost of a commercial gel electrophoresis apparatus including the gel-holding tray and the combs usually range from 500 USD to 2000 USD depending on the various models from different manufacturers (SigmaAldrich, ThermoFisher, Fisher Scientific,etc ) .

Table 1: Price obtained from related manufacturer's website.

\begin{tabular}{ll}
\hline Name/Type & Price (in USD) \\
\hline Thermo Scientific ${ }^{\mathrm{TM}}$ Owl & $\$ 688.32-\$ 837.80$ \\
EasyCast $^{\mathrm{TM}} \mathrm{B} 2 \mathrm{Mini}$ Gel & \\
FisherBiotech $^{\mathrm{TM}} \quad$ Horizontal & $\$ 312.64-\$ 1275.83$ \\
Electrophoresis Systems & \\
Thermo Scientific & \\
Invitrogen Owl $^{\mathrm{TM}}$ D4 & $\$ 1,155.29$ \\
\hline
\end{tabular}

\section{Conclusion}

We came to a solid conclusion based on the above observations that the unit with Chromium electrodes was quite effective for the normal use, with quality result and resolution of the bands at a lower cost compared to the commercially available units. This protocol upon optimization can be effectively embraced by the high schools, colleges and even universities to demonstrate the basic principle of gel electrophoresis and provide each student with an equal opportunity to get acquainted with the physical as well as working mechanisms of the gel electrophoresis equipment at a low cost.

From these observations, we verified that our unit is well suited for the demonstration of gelelectrophoresis procedures and applications in high school biology labs and even universities where each student may not have an equal access to the laboratory apparatus when mentored by their tutor due to its expensive nature, in a developing country like Nepal.

\section{References}

1. Ens S, Olson A, Dudley C, Ross N, Siddiqi A, Umoh $K$, Schneegurt $M$ : Inexpensive and safe DNA gel electrophoresis using household materials. Biochem Mol Biol Educ. 40(3), 198-203. doi:10.1002/bmb.20596

2. Lee P, Costumbrado J, Hsu C, Kim Y: Agarose Gel Electrophoresis for the Separation of DNA Fragments. J Visual Exp 2012.

3. Britos L, Goyenola G, Oroño S: Simple protocol for secondary school hands-on activity: Electrophoresis of pre-stained nucleic acids on agar-agar borate gels. Biochem $\mathrm{Mol}$ Biol Educ. 32(5), 341-347.
4. Green M, and Sambrook J: Molecular cloning: A laboratory manual 4th ed. Cold Spring Harbor: Cold Spring Harbor Laboratory Press. 2012

5. Sigmon J and Larcom L: The effect of ethidium bromide on mobility of DNA fragments in agarose gel electrophoresis. Electrophoresis 1996, 17:1524-1527.

6. InfoMine: Investment Mine. http://www. infomine.com/investment/ (2017). Accessed 10 June 2017

7. New England BioLabs: Product Catalog. https://www.neb.com/ products (2017). Accessed 10 June 2017.

8. Bancroft and Wilder D: Electrolytic Theory of Corrosion. J. Phys. Chem. 1924; DOI: $10.1021 / \mathrm{j} 150242 \mathrm{a} 001$

9. ImageJ: Image processing and Analysis in Java. https://imagej.nih.gov/ij/ (2004). Accessed 15 May 2016. 